\title{
Abdominal cocoon, a rare cause of intestinal obstruction: a case report and literature review
}

\author{
Alaa Sedik*, Mufid Maali, AbdelMajid EIShamary, Fahed EIShamary \\ Department of General Surgery, King Khalid Hospital Hail, Al Matar, Hail Saudi Arabia, Saudi Arabia
}

Received: 04 September 2017

Accepted: 28 September 2017

*Correspondence:

Dr. Alaa Sedik,

E-mail: asedik59@yahoo.com

Copyright: ( ) the author(s), publisher and licensee Medip Academy. This is an open-access article distributed under the terms of the Creative Commons Attribution Non-Commercial License, which permits unrestricted non-commercial use, distribution, and reproduction in any medium, provided the original work is properly cited.

\begin{abstract}
Abdominal cocoon syndrome or sclerosing encapsulating peritonitis is a rare condition that indicates that a part or whole small bowel is encased in a fibro collagenous membrane or cocoon leading to acute or chronic bowel obstruction. Approximately 50 cases have been reported in literature. Patients usually present with a picture of bowel obstruction and most cases are diagnosed during laparotomy. Herein, we present a 31 years old Indian male presented with symptoms and signs of acute small bowel obstruction that proved to be due to an abdominal cocoon intraoperatively. Our patient underwent resection with stapled side to side anastomosis. He was readmitted again with a single intraperitoneal collection; near the anastomotic site; that was managed percutaneously. He was discharged in a good condition and was seen later in the surgery clinic free of symptoms. Early diagnosis with radiological guidance and surgical intervention may reduce morbidity of the condition.
\end{abstract}

Keywords: Abdominal cocoon, Intestinal obstruction

\section{INTRODUCTION}

Abdominal cocoon is a rare condition that refers to a total or partial encapsulation of the small bowel by a fibro collagenous membrane or cocoon with local inflammatory reaction which leads to acute or chronic bowel obstruction. This was first described by Owtschinnikow, as 'Peritonitis chronic fibrosa incapsulata' and as 'sclerosing encapsulating peritonitis' by Deeb et al. ${ }^{1,2}$ Abdominal cocoon usually presents as acute or recurrent sub-acute intestinal obstruction with or without mass. Diagnosis of abdominal cocoon is usually made per operatively and surgical removal of obstruction causing membrane gives complete recovery.

\section{CASE REPORT}

32 years old Indian male; not known to have any medical problem before was admitted through emergency department with 3 days history of increasing abdominal pain and frequent vomiting followed by constipation and abdominal distension a day prior to presentation. He had constipation for 2 days but failed to flatus 1 day ago. No fever, jaundice, hematemesis or melena. He had less severe similar attacks that were treated conservatively.

Clinically, he was in severe pain, mildly dehydrated, afebrile with stable vital signs. Abdominal examination showed a distended rigid and generalized tender abdomen with exaggerated bowel sounds. No masses could be palpated due to pain. Rectum was empty. Resuscitation was started with IV lactated ringer solution, $\mathrm{O}_{2}$ mask, empiric antibiotics, Nasogastric tube and Foley's catheter were inserted to monitor fluid status. Lab works showed that WBC 20,000; little renal function impairment; ABG showed metabolic acidosis. His coagulation profile was little impaired. Plain X-rays abdomen showed distended bowel loops with multiple air-fluid levels with absence of 
gas in the rectum; suggestive of complete small bowel obstruction. (Figure 1) abdominal ultrasonography (US) was inconclusive.

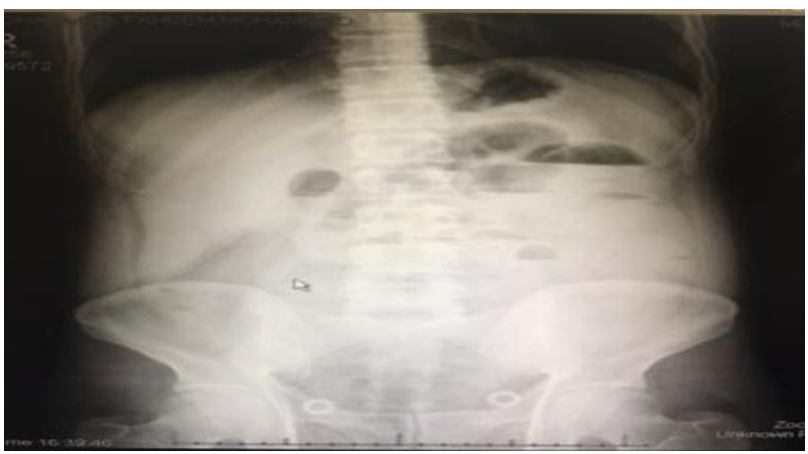

Figure 1: Multiple air fluid levels with absence of gas in the rectum; suggestive findings of complete small bowel obstruction.

Contrast enhanced abdominal computerized scan (CECT) was not done due to the renal impairment. Patent was prepared and consented for laparotomy with possible bowel resection and or stoma formation. The abdomen was entered and most of the distal terminal ileum just proximal to the ileocecal valve was encapsulated or encased in a very tough whitish membrane (Figure 2, a). There was a $1 \mathrm{~cm}$ hard yellowish beans-like stone?? found free intra-peritoneally; with no evidence of bowel perforation. There were dense adhesions between the liver and abdominal wall all around the liver and to the stomach and also to the area of duodenum. Trials of adhesiolysis were failed with evidence of vascular compromise of the affected friable bowel. The proximal part of the ascending colon, cecum, and most of the affected terminal ileum was resected and continuity restored by stapled side to side anastomosis (Figure 2, b).

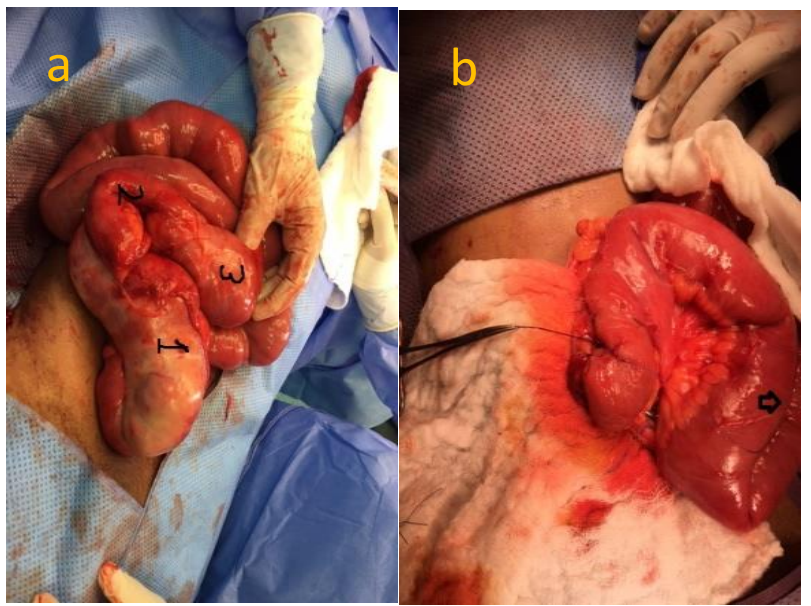

Figure 2: Intraoperative findings, (a) Abdominal cocoon (1) after trial of adhesiolysis, (2) that failed to be completed safely from the region of the cecum, (3) Multiple patches of gangrene (not shown here) were found, (b) the affected areas were resected; then stapled anastomosis done.
The abdomen was washed with saline and 2 drains were left (abdominal and pelvic); then closed with mass closure technique. The patient had uneventful recovery and discharged on the $10^{\text {th }}$ postoperative day in good condition.

Two weeks later, he came to OPD for follow-up where he was admitted again for mild right lumbar pain and ?? leak from the abdominal drain site in this area. There was no fever, passing normal motions and tolerating full diet.

Clinically, afebrile with stable vital signs. He was not in pain. The abdomen was soft mildly tender around the drain site with deep mildly tender $4 \times 5 \mathrm{~cm}$ palpable mass underneath. Culture and sensitivity (C and $\mathrm{S}$ ) was taken from the purulent discharge coming from the drain site. There was a superficial wound infection at the region of umbilicus with clips were still in place. All clips were removed wound opened and $\mathrm{C}$ and $\mathrm{S}$ taken from the discharge. He was kept Nil per OS, IV fluids, antibiotics, SC enoxaparin. Lab-works showed WBC of 18,000 . U S was unremarkable; except for a right lumbar hypo-echoic collection of $5 \times 6 \mathrm{~cm}$ with no free intra-peritoneal fluid.

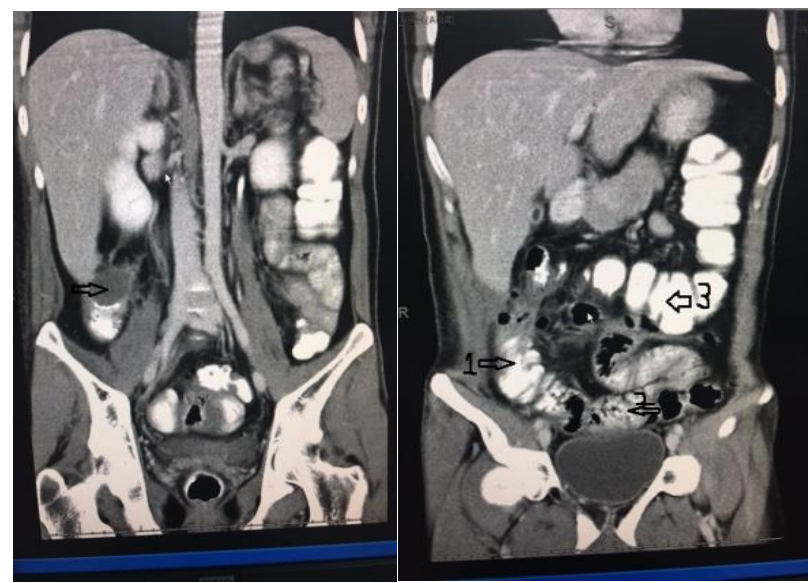

Figure 3: (a) CECT- the intra-peritoneal collection without evidence of leaks. (b) showed the flow of contrast from the ilium (arrow 2) to the distal ascending colon (arrow 1); going distal to the transverse and descending colon (arrow3).

CECT was done and confirmed the previous US findings with no definite leaks (Figure 3). Patient was offered percutaneous US- guided catheter (Gauge 12) drainage of the collection. His WBC came down to normal in 3 days. After 5 days, the catheter drainage became nil, a follow up US showed only a very minimal collection could be seen. The catheter was removed and patient discharged for OPD follow-up. He was ok when he was seen in OPD after 3 weeks.

\section{DISCUSSION}

Abdominal cocoon or sclerosing encapsulating peritonitis (SEP) is a rare condition that refers to total or partial encapsulation of the small bowel by a fibro collagenous 
membrane or cocoon with local inflammatory infiltrate leading to acute or chronic bowel obstruction. ${ }^{1}$ The abdominal cocoon was first described and named in 1978 by Foo et al. Since then, approximately 50 cases have been reported in literature. . $^{2,3}$

The majority of the cases are reported from tropical and subtropical climate belts of the world. The only case reported from a non-tropical zone is from England, in which the case was born in Pakistan. The SEP is mainly an acquired disease. Many causes were listed in the pathogenesis of SEP including previous abdominal surgery or peritonitis, chronic ambulatory peritoneal dialysis and prolonged use of drug:practolol.

Other conditions have been mentioned; including ventriculo-peritoneal and peritoneo-venous shunts, SLE, sarcoidosis, cirrhosis, tuberculosis, propranolol use for constrictive pericarditis, uterine and ovarian tumors, and recurrent peritonitis. ${ }^{4-6}$ Clinically, most patients with abdominal cocoon syndrome (ACS) present with features of recurrent acute or chronic small bowel obstruction secondary to kinking and/or compression of the intestines within the constricting cocoon. ${ }^{3}$ Yip and Lee listed four main clinical features that help to identify abdominal cocoon pre-operatively including: young girl with no other cause of bowel obstruction, similar attacks that resolved spontaneously, abdominal pain and vomiting, and a nontender soft abdominal mass on palpation. Abdominal X-ray and US are not useful to differentiate ACS from other causes of bowel obstruction. ${ }^{7}$ CECT is more beneficial in differential diagnosis. The finding of a fibrous membrane enclosing the intestinal loops is a typical radiological finding of ACS. However, although radiological findings, especially computed tomography, are helpful; yet, final and definite diagnosis is generally by surgery, as confirmed by ACS literature. ${ }^{7-9}$ Treatment consists of excision of the peritoneal sac with lysis of the interloop adhesions. Bowel resection is done if a nonviable segment is found. ${ }^{4}$

\section{CONCLUSION}

Abdominal cocoon syndrome is rare and difficult to diagnose condition which requires better awareness of the condition. A combination of clinical examination and radiologic studies may help to diagnose it preoperatively. Early presentation and intervention can improve the outcome of this rare condition.

Funding: No funding sources Conflict of interest: None declared

Ethical approval: Not required

\section{REFERENCES}

1. Owtschinnikow PJ. Peritonitis chronic fibrosa incapsulata. ArchKlin Chir. 1907;83:623-4.

2. Constantinides F, DiNunno N, Bernasconi P, Jonjic $\mathrm{N}$, Melato M. A new type of peritoneal encapsulation of the small bowel. Am J Surg Pathol. 1998;22(10):1297-8. [PubMed]

3. Deeb LS, Mourad FH, El-Zein YR, Uthman SM. Abdominal cocoon in a man: preoperative diagnosis and literature review. J Clin Gastroenterol. 1998;26(2):148-50. [PubMed]

4. Foo KT, Ng KC, Rauff A, Foong WC, Sinniah R. Unusual small intestinal obstruction in adolescent girls: the abdominal cocoon. $\mathrm{Br} \mathrm{J}$ Surg. 1978;65(6):427-30.

5. Kaushik R, Punia RPS, Mohan H, Attri AK. Case report. Tuberculous abdominal cocoon- a report of 6 cases and review of literature. W J Emergency Surg. 2006;1:18.

6. Hasan MFM, Muhsin OMA, Abassi AA, Youssufani R. Abdominal Cocoon: A report of three cases. Middle East J Emergency Med. 2007;7(2). Available http://www.hmc.org.qa/mejem/sept2007/Edited/ case 2.htm.

7. Kaushik R, Punia RP, Mohan H, Attri AK. Tuberculous abdominal cocoon--a report of 6 cases and review of the Literature. World J Emerg Surg. 2006;27:1:18.

8. Yip FW, Lee SH. The abdominal cocoon. Aust N Z J Surg. 1992;62(8):638-42.

9. Gupta S, Shirahatti RG, Anand J. CT findings of an abdominal cocoon. AJR Am J Roentgenol. 2004;183(6):1658-60.

10. Demir MK, Akinci O, Onur E, Koksal N. Case 108: Sclerosing Encapsulating Peritonitis. Radiol. 2007;242:937-9.

11. Hosein HH, Quane LK, Cohen AJ. Abdominal cocoon. Applied Radiology 2003;32(10). Available at http://www.medscape.com/viewarticle/464849_prin.

Cite this article as: Sedik A, Maali M, ElShamary A, ElShamary F. Abdominal cocoon, a rare cause of intestinal obstruction: a case report and literature review. Int Surg J 2017;4:3753-5. 